\section{Hydrogel-nanoparticle hybrids for biomedical applications: principles and advantages}

By: Sulaiman, NS (Sulaiman, Nur Shahirah) ${ }^{1}$; Hamzah, N (Hamzah, Nurasyikin) ${ }^{2}$; Zakaria, SF (Zakaria, Siti Fatimah) ${ }^{3}$; Othman, SFC (Che Othman, Siti Fairuz) ${ }^{4}$; Suffian, IFM (Mohamed Suffian, Izzat Fahimuddin) ${ }^{1}$ View Web of Science ResearcherID and ORCID (provided by Clarivate)

NANOMEDICINE

Volume: 16 Issue: 2 Page: $81-84$

DOI: $10.2217 / \mathrm{nnm}-2020-0420$

Published: NOV 2020

Early Access: DEC 2020

Document Type: Editorial Material

Keywords

Author Keywords: biomedical; hybrid; hydrogel; nanoparticle-hydrogel hybrid; nanoparticles; treatment

Keywords Plus: LOADED NANOCAPSULES; DELIVERY; FORMULATION; SYSTEMS

Author Information

Corresponding Address: Mohamed Suffian, Izzat Fahimuddin (corresponding author)

- Int Islamic Univ Malaysia, Dept Pharmaceut Chem, Kulliyyah Pharm, Kuantan 25200, Pahang, Malaysia

Addresses:

- ${ }^{1}$ Int Islamic Univ Malaysia, Dept Pharmaceut Chem, Kulliyyah Pharm, Kuantan 25200, Pahang, Malaysia

$\checkmark 2$ Int Islamic Univ Malaysia, Dept Chem, Kulliyyah Sci, Kuantan 25200, Pahang, Malaysia

- 3 Int Islamic Univ Malaysia, Dept Computat \& Theoret Sci, Kulliyyah Sci, Kuantan 25200, Pahang, Malaysia

$\checkmark{ }^{4}$ Int Islamic Univ Malaysia, Dept Biotechnol, Kulliyyah Sci, Kuantan 25200, Pahang, Malaysia

E-mail Addresses: izzat_fahimuddin@iium.edu.my

\section{Categories/Classification}

Research Areas: Biotechnology \& Applied Microbiology; Science \& Technology - Other Topics Funding

\begin{tabular}{|ll|}
\hline Funding agency & Grant number \\
Fundamental Research Grant Scheme for Research Acculturation of Early & RACER19-022- \\
Career Researchers & 0022 \\
& RIGS17-044- \\
Research Initiative Grant Scheme & 0619 \\
& RIGS17-050- \\
\hline
\end{tabular}

View funding text

\section{+ See more data fields}

\section{Journal information}

Nanomedicine

ISSN: $1743-5889$

elSSN: $1748-6963$

Current Publisher: FUTURE MEDICINE LTD, UNITEC HOUSE, 3RD FLOOR, 2 ALBERT

Journal Impact Factor: Journal Citation Report ${ }^{\mathrm{TM}}$

Research Areas: Biotechnology \& Applied Microbiology; Science \& Technology - Other Topics

\section{Citation Network}

In Web of Science Core Collection

0

Citations

Create citation alert

Cited References

\section{6}

View Related Records

You may also like...

Song, P; Ye, DK; Zuo, XL; et al.

Preparation and Biological Applications of DNA Hydrogel

PROGRESS IN CHEMISTRY

Kim, BS; Cho, CS;

Injectable Hydrogels for Regenerative Medicine TISSUE ENGINEERING AND REGENERATIVE MEDICINE

Ji, Q; Zhang, H; Qiu, LP; et al.

Hydrosoluble collagen based biodegradable hybrid hydrogel for biomedical scaffold JOURNAL OF BIOMATERIALS SCIENCEPOLYMER EDITION

Laftah, WA; Hashim, S; Ibrahim, AN; Polymer Hydrogels: A Review POLYMER-PLASTICS TECHNOLOGY AND ENGINEERING

Wang, LY; Huang, J; Mao, H; et al. Exerting Enhanced Permeability and Retention Effect Driven Delivery by Ultrafine Iron Oxide Nanoparticles with T-1-T-2 Switchable Magnetic Resonance Imaging Contrast ACS NANO

See all

\section{Use in Web of Science}

Web of Science Usage Count

\section{5} 15

Last 180 Days Since 2013

Learn more

This record is from:

Web of Science Core Collection

Science Citation Index Expanded 
Web of Science Categories: Biotechnology \& Applied Microbiology; Nanoscience \& Nanotechnology

26 Cited References

Showing 26 of $26 \quad$ VIEW AS SET OF RESULTS

(from Web of Science Core Collection) 
